\title{
CAS Mathematica in Random Studies
}

\author{
Igor E. Poloskov \\ Perm State University, Bukirev st., 15, 614990, Perm, GSP, Russia \\ Igor.Poloskov@psu.ru
}

\begin{abstract}
It is known that a lot of mathematical calculations must be processed during a practical use of exact and approximate methods, which are designed to analyze random phenomena in nonlinear dynamic systems. The present paper describes some schemes of such analysis. These schemes expect an application of a computer algebra system (CAS) Mathematica v. 4.1 [9], which is a very powerful and suitable tool for above mentioned topic. At first, relations between moments, cumulants, and quasi-moments are derived. Further, a problem concerning the seach of polynomial stochastic potentials is considered. Necessary conditions and relations are obtained to solve the last problem. All calculations of appropriate expressions were produced with the help of our package ProbRel and different procedures.
\end{abstract}

\section{Introduction}

The analysis of random processes in nonlinear dynamic systems is a very important topic for theory and practice. The necessity of such analysis is urgent for studying various phenomena: (i) a flight of vehicles under an action of atmospheric turbulence; (ii) traffic on a rough surface; (iii) high-altitude vibrations of structures under wind and seismic attacks; (iv) a rolling of ships due to a rough-sea etc.

Solving a significant number of practical problems, it is possible to assume that a random vector process $\boldsymbol{x} \in \mathbf{X} \subset \mathbf{R}^{n}$ describing a status of an object being studied, a phase vector, satisfies a set of stochastic differential equations (SD Eqs.) in the Stratonovich sense [1/2]

$$
d \boldsymbol{x}(t)=\boldsymbol{f}(\boldsymbol{x}, t) d t+G(\boldsymbol{x}, t) d \boldsymbol{w}(t),
$$

where $\dot{\boldsymbol{w}}=\boldsymbol{\xi} \in \mathbf{R}^{m}$ is a vector of independent Gaussian white noises with unit intensities; $\boldsymbol{w} \in \mathbf{R}^{m}$ is the standardized Wiener vector process; $\boldsymbol{f}(\cdot, \cdot)=$ $\left\{f_{i}(\cdot, \cdot)\right\}^{T}: \mathbf{R}^{n} \times[0, \infty) \rightarrow \mathbf{R}^{n}$ is a deterministic vector-function; $G(\cdot, \cdot)=$ $\left\{g_{i j}(\cdot, \cdot)\right\}: \mathbf{R}^{n} \times[0, \infty) \rightarrow \mathbf{R}^{n} \times \mathbf{R}^{m}$ is a deterministic matrix-function; $\mathrm{T}$ is a symbol of the transposition.

The main stochastic characteristics of the vector $\boldsymbol{x}$ are the probability density function (PDF) $p(\boldsymbol{x}, t)$; the transition probability density function(TPDF) $p(\boldsymbol{x}, t \mid \boldsymbol{y}, \tau)$; the characteristic function $\Theta(\boldsymbol{\lambda}, t) ;$ moments $m_{\alpha}=\mathbf{M}\left[x^{\alpha}\right]$ and 
$\mu_{\alpha}=\mathbf{M}\left[(x-\mathbf{M}[x])^{\alpha}\right]$, where $\alpha=\left\{\alpha_{1}, \alpha_{2}, \ldots, \alpha_{n}\right\}$ is a multi-index, $\alpha_{i} \geq 0$; cumulants $\varkappa_{\alpha}$; covariance functions $\mathcal{K}_{i j}\left(t_{1}, t_{2}\right)$, and spectral density functions $s_{i j}(\omega)$. Among moments the first ones are highlighted such as mean values $m_{i}=\left\langle x_{i}\right\rangle$; variances $\sigma_{i}^{2}$; mixed moments $m_{i j}$ and $\mu_{i j}$ of second order. In the case $t_{1}=t_{2}=t$ we denote $\mathcal{K}_{i j}$ as $\mathcal{D}_{i j}(t)$, where $\mathcal{D}_{i j}=\mathbf{M}\left[\left(x_{i}-m_{i}\right)\left(x_{j}-m_{j}\right)\right]$ are elements of the matrix $\mathcal{D}$ of covariances, and $\mathbf{M}[\cdot]$ stands for the mathematical expectation.

It is well known 112 that the TPDF $p(\boldsymbol{x}, t \mid \boldsymbol{y}, \tau)$ satisfies the Fokker - Planck - Kolmogorov equation (FPK Eq.)

$$
\frac{\partial p(\boldsymbol{x}, t \mid \boldsymbol{y}, \tau)}{\partial t}=\mathbf{L}_{x t} p(\boldsymbol{x}, t \mid \boldsymbol{y}, \tau), \quad \lim _{t \rightarrow \tau+0} p(\boldsymbol{x}, t \mid \boldsymbol{y}, \tau)=\delta(\boldsymbol{x}-\boldsymbol{y}) .
$$

The operator $\mathbf{L}$ within Eq. (1.2) has the form

$$
\mathbf{L}_{x t} v=-\sum_{i=1}^{n} \frac{\partial}{\partial x_{i}}\left[a_{i}(\boldsymbol{x}, t) v\right]+\frac{1}{2} \sum_{i, j=1}^{n} \frac{\partial^{2}}{\partial x_{i} \partial x_{j}}\left[b_{i j}(\boldsymbol{x}, t) v\right]
$$

where

$$
a_{i}=f_{i}+\frac{1}{2} \sum_{j=1}^{n} \sum_{k=1}^{m} \frac{\partial g_{i k}}{\partial x_{j}} g_{j k}, \quad B \equiv\left\{b_{i j}\right\}=G G^{T} .
$$

The PDF $p(\boldsymbol{x}, t)$ satisfies Eq.(1.2) too but with the initial condition $p\left(\boldsymbol{x}, t_{0}\right)=$ $p_{0}(\boldsymbol{x})$.

If $t$ tends to infinity, then under certain conditions there is a steady-state PDF $p_{s}(\boldsymbol{x})$, which satisfies the stationary FPK Eq.

$$
\mathbf{L} p_{s}(\boldsymbol{x})=0, \quad \boldsymbol{a}=\boldsymbol{a}(\boldsymbol{x}), \quad B=B(\boldsymbol{x}) .
$$

This solution does not depend on the initial distribution.

In addition, it is possible to write the equation to find the stochastic potential $\phi(\boldsymbol{x}, t)=-\ln p(\boldsymbol{x}, t)$

$$
\frac{\partial \phi}{\partial t}=\frac{1}{2} \sum_{i, j=1}^{n} \mathrm{~A}_{i j}\left(\frac{\partial^{2} \phi}{\partial x_{i} \partial x_{j}}-\frac{\partial \phi}{\partial x_{i}} \frac{\partial \phi}{\partial x_{j}}\right)+\sum_{i=1}^{n} \mathrm{~B}_{i} \frac{\partial \phi}{\partial x_{i}}-\mathrm{C} \equiv \tilde{\mathcal{K}}[\phi],
$$

where

$$
\mathrm{A}_{i j}=b_{i j}, \quad \mathrm{~B}_{i}=-a_{i}+\sum_{j=1}^{n} \frac{\partial b_{i j}}{\partial x_{j}}, \quad \mathrm{C}=\sum_{i=1}^{n} \frac{\partial}{\partial x_{i}}\left(-a_{i}+\frac{1}{2} \sum_{j=1}^{n} \frac{\partial b_{i j}}{\partial x_{j}}\right) .
$$

Nowadays there is a significant number of exact and approximate methods intended for an analysis of random phenomena. But to perform most of them, time consuming mathematical calculations such as transformations, reductions of similar terms, differentiations, integrations etc., are required. Computer algebra is a well-known tool for different sciences. Below we demonstrate an application of the package Mathematica for algebraic manipulations during a solution of some random study problems. 


\section{Relations for Characteristics}

An application of different probability methods demands very often to use some characteristics of random variables such as moments, cumulants, and quasimoments. For example, these characteristics are used to neglect infinite sets of OD Eqs. for moments during an exploration of nonlinear dynamical systems effected by random fluctuations if the quasi-Gaussian or cumulant closure is exploited. In practice, it is necessary to know simple relations among above mentioned values. A construction of relations for mixed moments, cumulants, and quasi-moments is considered in this section.

\subsection{Building of Relations}

To derive the dependencies, we use the technique from Ref. [7. Suppose all required characteristics of a random vector $\boldsymbol{x}$ exist and formal manipulations with power series are permissible.

Below we use the following multi-index notation:

$$
\begin{gathered}
\alpha=\left\{\alpha_{1}, \alpha_{2}, \ldots, \alpha_{n}\right\}, \quad \beta=\left\{\beta_{1}, \beta_{2}, \ldots, \beta_{n}\right\} \\
k_{1} \alpha+k_{2} \beta=\left\{k_{1} \alpha_{1}+k_{2} \beta_{1}, \ldots, k_{1} \alpha_{n}+k_{2} \beta_{n}\right\} \\
e_{k}=\left\{\delta_{k j}\right\}, \quad j=1,2, \ldots, n, \quad e_{k j}=e_{k}+e_{j}, \\
x^{\alpha}=x_{1}^{\alpha_{1}} x_{2}^{\alpha_{2}} \ldots x_{n}^{\alpha_{n}}, \quad \mathrm{C}_{\alpha}^{\beta}=\mathrm{C}_{\alpha_{1}}^{\beta_{1}} \mathrm{C}_{\alpha_{2}}^{\beta_{2}} \ldots \mathrm{C}_{\alpha_{n}}^{\beta_{n}}, \\
\sum_{\beta=0}^{\alpha} h_{\beta}=\sum_{\beta_{1}=0}^{\alpha_{1}} \sum_{\beta_{2}=0}^{\alpha_{2}} \ldots \sum_{\beta_{n}=0}^{\alpha_{n}} h_{\beta_{1} \beta_{2} \ldots \beta_{n}}, \quad|\beta|=\beta_{1}+\beta_{2}+\ldots+\beta_{n} .
\end{gathered}
$$

Here $\delta_{k j}$ is the Kronecker delta; $C_{s}^{q}$ is equal to $s ! / q !(s-q) !$.

In addition, let $\boldsymbol{x}=\left\{x_{1}, x_{2}, \ldots, x_{n}\right\}$ be a random vector, $p(\boldsymbol{x})$ be the probability density of $\boldsymbol{x}$, and $\Theta(\boldsymbol{u})$ be the characteristic function:

$$
\Theta(\boldsymbol{u})=\mathbf{M}\left[\exp \left(i \sum_{k=1}^{n} x_{i} u_{i}\right)\right]
$$

where $i$ is equal to $\sqrt{-1} ; \boldsymbol{u}=\left\{u_{1}, u_{2}, \ldots, u_{n}\right\}$.

Transforming and generalizing relations from Refs.[348], we get the function $\Theta(\boldsymbol{u})$ in the following forms:

$$
\begin{gathered}
\Theta(\boldsymbol{u})=\sum_{\alpha=0}^{\infty} \frac{i^{|\alpha|}}{\alpha !} m_{\alpha} u^{\alpha}, \\
\Theta(\boldsymbol{u})=\exp \left[\sum_{\alpha=0}^{\infty} \frac{i^{|\alpha|}}{\alpha !} \varkappa_{\alpha} u^{\alpha}\right], \\
\Theta(\boldsymbol{u})=\exp \left[i \sum_{j=1}^{n} a_{j} u_{j}-\frac{1}{2} \sum_{j, k=1}^{n} \mathcal{D}_{j k} u_{j} u_{k}\right] \sum_{\alpha=0}^{\infty} \frac{i^{|\alpha|}}{\alpha !} q_{\alpha} u^{\alpha} .
\end{gathered}
$$

Here $m_{\lambda}, \varkappa_{\lambda}, q_{\lambda}$ are mixed moments, cumulants, and quasi-moments of the random vector $\boldsymbol{x} ; m_{0}=1 ; \varkappa_{0}=0 ; q_{0}=1$. 
Lemma. The relations connecting mixed moments and cumulants of a random vector are

$$
\begin{gathered}
m_{\alpha+e_{s}}=\sum_{\beta=0}^{\alpha} \mathrm{C}_{\alpha}^{\beta} \varkappa_{\beta+e_{s}} m_{\alpha-\beta}, \\
\varkappa_{\alpha+e_{s}}=m_{\alpha+e_{s}}-\sum_{\beta=0, \beta \neq \alpha}^{\alpha} \mathrm{C}_{\alpha}^{\beta} \varkappa_{\beta+e_{s}} m_{\alpha-\beta}, \quad \alpha \geq 0,
\end{gathered}
$$

where $s$ is an integer and $1 \leq s \leq n$.

Proof. Let's make use of notations (2.1) and relations (2.2), (2.3). If we differentiate both sides of last relations with respect to $u_{s}, 1 \leq s \leq n$, then we obtain

$$
\begin{gathered}
\frac{\partial \Theta(\boldsymbol{u})}{\partial u_{s}}=\sum_{\alpha=0}^{\infty} \frac{i^{|\alpha|}}{\alpha !} \alpha_{s} m_{\alpha} u^{\alpha-e_{s}}=\sum_{\alpha=0}^{\infty} \frac{i^{\left|\alpha+e_{s}\right|}}{\alpha !} m_{\alpha+e_{s}} u^{\alpha} \\
\frac{\partial \Theta(\boldsymbol{u})}{\partial u_{s}}=\Theta(\boldsymbol{u}) \cdot \sum_{\alpha=0}^{\infty} \frac{i^{\left|\alpha+e_{s}\right|}}{\alpha !} \varkappa_{\alpha+e_{s}} u^{\alpha}=\sum_{\alpha=0}^{\infty} \frac{i^{|\alpha|}}{\alpha !} m_{\alpha} u^{\alpha} \cdot \sum_{\alpha=0}^{\infty} \frac{i^{\left|\alpha+e_{s}\right|}}{\alpha !} \varkappa_{\alpha+e_{s}} u^{\alpha}= \\
=\sum_{\alpha=0}^{\infty} \frac{i^{\left|\alpha+e_{s}\right|}}{\alpha !} u^{\alpha} \sum_{\beta=0}^{\alpha} \mathrm{C}_{\alpha}^{\beta} \varkappa_{\beta+e_{s}} m_{\alpha-\beta}
\end{gathered}
$$

Equating the right-hand sides of relations (2.7), (2.8) and selecting the coefficients of terms $i^{\left|\alpha+e_{s}\right|} u^{\alpha} / \alpha$ !, we derive the desired dependencies (2.5) and (2.6).

Corollary 1. Senior mixed moments of the $n$-dimensional Gaussian distribution

$$
p(\boldsymbol{x}) \equiv \mathcal{N}(\langle\boldsymbol{x}\rangle, \mathcal{D})=\frac{1}{\sqrt{(2 \pi)^{n}|\mathcal{D}|}} \exp \left[-\frac{1}{2} \sum_{j, k=1}^{n} \mathcal{D}_{j k}^{-1}\left(x_{j}-\left\langle x_{j}\right\rangle\right)\left(x_{k}-\left\langle x_{k}\right\rangle\right)\right]
$$

could be calculated as follows

$$
m_{\alpha}=\left\langle x_{s}\right\rangle m_{\alpha-e_{s}}+\left(\alpha_{s}-1\right) \mathcal{D}_{s s} m_{\alpha-2 e_{s}}+\sum_{k=1, k \neq s}^{n} \alpha_{k} \mathcal{D}_{s k} m_{\alpha-e_{s k}},
$$

where $|\alpha|>0,|\mathcal{D}|$ is the determinant of matrix $\mathcal{D}, \mathcal{D}_{j k}^{-1}$ are elements of inverse matrix for the matrix $\mathcal{D}$ and $s$ is any integer from the interval $1 \leq s \leq n$.

Proof. Let's take into account the equalities

$$
\begin{gathered}
m_{e_{s}}=\varkappa_{e_{s}}=\left\langle x_{s}\right\rangle, \quad \varkappa_{e_{s k}}=\mathcal{D}_{s k}, \\
m_{e_{s k}}=\varkappa_{e_{s k}}+\varkappa_{e_{s}} \varkappa_{e_{k}}=\mathcal{D}_{s k}+\left\langle x_{s}\right\rangle\left\langle x_{k}\right\rangle, \\
\varkappa_{\alpha}=0, \quad \alpha \neq e_{s}, e_{s k}, \quad s, k=\overline{1, n} .
\end{gathered}
$$

Then using (2.5) and (2.10), we get

$$
m_{\alpha+e_{s}}=m_{\alpha} \varkappa_{e_{s}}+\sum_{k=1}^{n} \alpha_{k} m_{\alpha-e_{k}} \varkappa_{e_{s k}}=\left\langle x_{s}\right\rangle m_{\alpha}+\sum_{k=1}^{n} \alpha_{k} \mathcal{D}_{s k} m_{\alpha-e_{k}} .
$$

The desired relation (2.9) is obtained from this equality after a shift of index. 
Corollary 2. Moments and quasi-moments of random vector $\boldsymbol{x}$ are connected as follows

$$
m_{\alpha}=\sum_{\beta=0}^{\alpha} \mathrm{C}_{\alpha}^{\beta} m_{\alpha-\beta}^{\mathcal{N}} q_{\beta}
$$

where the moments $m_{\lambda}^{\mathcal{N}}$ may be calculated by formulae $(2.9)$.

Proof. Let's consider expression (2.4). The first multiplier in the right-hand side represents the characteristic function of $n$-dimensional Gaussian distribution. Hence by (2.2), this multiplier may be represented as

$$
\exp \left[i \sum_{j=1}^{n}\left\langle x_{j}\right\rangle u_{j}-\frac{1}{2} \sum_{j, k=1}^{n} \mathcal{D}_{j k} u_{j} u_{k}\right]=\sum_{\alpha=0}^{\infty} \frac{i^{|\alpha|}}{\alpha !} m_{\alpha}^{\mathcal{N}} u^{\alpha} .
$$

Thus an expression for the characteristic function $\Theta(\boldsymbol{u})$ may be written down in the form of

$$
\Theta(\boldsymbol{u})=\sum_{\alpha=0}^{\infty} \frac{i^{|\alpha|}}{\alpha !} m_{\alpha}^{\mathcal{N}} u^{\alpha} \cdot \sum_{\alpha=0}^{\infty} \frac{i^{|\alpha|}}{\alpha !} q_{\alpha} u^{\alpha} .
$$

Multiplying two series in the right-hand side of the last equality, we obtain

$$
\Theta(\boldsymbol{u})=\sum_{\alpha=0}^{\infty} \frac{i^{|\alpha|}}{\alpha !} u^{\alpha} \sum_{\beta=0}^{\alpha} \mathrm{C}_{\alpha}^{\beta} m_{\alpha-\beta}^{\mathcal{N}} q_{\beta}
$$

Relation (2.11) will be derived if we equate the coefficients of $i^{|\alpha|} u^{\alpha} / \alpha$ ! in the right-hand sides of equalities (2.2) and (2.12).

Corollary 3. Cumulants and quasi-moments of random vector $\boldsymbol{x}$ are connected as follows

$$
\sum_{\beta=0}^{\alpha+e_{s}} \mathrm{C}_{\alpha+e_{s}}^{\beta} m_{\alpha-\beta+e_{s}}^{\mathcal{N}} q_{\beta}=\sum_{\gamma=0}^{\alpha} \mathrm{C}_{\alpha}^{\gamma} \varkappa_{\alpha-\gamma+e_{s}} \sum_{\beta=0}^{\gamma} \mathrm{C}_{\gamma}^{\beta} m_{\gamma-\beta}^{\mathcal{N}} q_{\beta}
$$

Proof will be trivial if we use calculations being performed under the proof of Lemma and Corollary 2.

If it is necessary, then using (2.13), quasi-moments may be derived from cumulants and vice versa just the same way as relations (2.5) and (2.6).

In the case of $n=1$ relations $(2.5),(2.6),(2.9),(2.11),(2.13)$ take the form

$$
\begin{aligned}
m_{s+1} & =\sum_{k=0}^{s} \mathrm{C}_{s}^{k} \varkappa_{k+1} m_{s-k}, \quad \varkappa_{s+1}=m_{s+1}-\sum_{k=0}^{s-1} \mathrm{C}_{s}^{k} \varkappa_{k+1} m_{s-k}, \\
m_{s} & =\langle x\rangle m_{s-1}+(s-1) \sigma^{2} m_{s-2}, \quad m_{s}=\sum_{k=0}^{s} \mathrm{C}_{s}^{k} m_{s-k}^{\mathcal{N}} q_{k} \\
& \sum_{k=0}^{s+1} \mathrm{C}_{s+1}^{k} m_{s-k+1}^{\mathcal{N}} q_{k}=\sum_{r=0}^{s} \mathrm{C}_{s}^{r} \varkappa_{s-r+1} \sum_{k=0}^{r} \mathrm{C}_{r}^{k} m_{r-k}^{\mathcal{N}} q_{k} .
\end{aligned}
$$




\subsection{Application of Relations}

To use in practice the relations derived, a Mathematica code package ProbRels has been implemented. Some aims of this package construction are the following:

(i) to calculate an indefinite integral, where the integrant is a product of a multi-dimensional Gaussian probability density and polynomial, in a symbolic mode by computer. For example, such integration is necessary for realization of the Gaussian approximation method for a stochastic analysis of nonlinear dynamic systems;

(ii) to derive relations for the quasi-Gaussian and cumulant closure in above mentioned analysis.

Below we demonstrate the use of the package. Here there are Mathematica commands for calls of procedures from the package and results of calculations.

Example 2.1. If we need to find the mean value of the expression $3 x_{1}^{2} x_{2}+$ $4 x_{1} x_{2} x_{3}-x_{1}^{5} x_{3}$, where $\left\{x_{1}, x_{2}, x_{3}\right\}$ is a vector with a Gaussian distribution, then we may call the IntGauss procedure as

$$
\begin{gathered}
\text { IntGauss }\left[\left\{\mathrm{x}_{1}, \mathrm{x}_{2}, \mathrm{x}_{3}\right\}, 3 \mathrm{x}_{1}^{2} \mathrm{x}_{2}+4 \mathrm{x}_{1} \mathrm{x}_{2} \mathrm{x}_{3}-\mathrm{x}_{1}^{5} \mathrm{x}_{3},\left\{\mathrm{~m}_{1}, \mathrm{~m}_{2}, \mathrm{~m}_{3}\right\}\right. \\
\left.\left\{\left\{\mathrm{d}_{11}, \mathrm{~d}_{12}, \mathrm{~d}_{13}\right\},\left\{\mathrm{d}_{21}, \mathrm{~d}_{22}, \mathrm{~d}_{23}\right\},\left\{\mathrm{d}_{31}, \mathrm{~d}_{32}, \mathrm{~d}_{33}\right\}\right\}\right]
\end{gathered}
$$

where the third parameter of the procedure is the vector of mean values and the forth one is the matrix of covariances. The result is

$$
\begin{gathered}
-15 d_{11}^{2} d_{13}+6 d_{12} m_{1}+4 d_{23} m_{1}-30 d_{11} d_{13} m_{1}^{2} \\
-5 d_{13} m_{1}^{4}+3 d_{11} m_{2}+4 d_{13} m_{2}+3 m_{1}^{2} m_{2} \\
+4 d_{12} m_{3}-15 d_{11}^{2} m_{1} m_{3}-10 d_{11} m_{1}^{3} m_{3}-m_{1}^{5} m_{3}+4 m_{1} m_{2} m_{3}
\end{gathered}
$$

Note we have taken into account here that the matrix of covariances is a symmetric one.

Example 2.2. If we need to produce the Gaussian closure of the expression $3 x_{1}^{2} x_{2}+4 x_{1} x_{2} x_{3}-x_{1}^{5} x_{3}$ to exclude moments with orders more than 3 , where $\left\{x_{1}, x_{2}, x_{3}\right\}$ is a vector with the vector $\alpha$ of mean values and the matrix $\left\{d_{i j}\right\}$ of covariances, then we may call the QuasiGauss procedure as

$$
\begin{gathered}
\text { QuasiGauss }\left[\left\{\mathrm{x}_{1}, \mathrm{x}_{2}, \mathrm{x}_{3}\right\}, 3 \mathrm{x}_{1}^{2} \mathrm{x}_{2}+4 \mathrm{x}_{1} \mathrm{x}_{2} \mathrm{x}_{3}-\mathrm{x}_{1}^{5} \mathrm{x}_{3},\right. \\
\left.\left\{\mathrm{m}_{1}, \mathrm{~m}_{2}, \mathrm{~m}_{3}\right\},\left\{\left\{\mathrm{d}_{11}, \mathrm{~d}_{12}, \mathrm{~d}_{13}\right\},\left\{\mathrm{d}_{21}, \mathrm{~d}_{22}, \mathrm{~d}_{23}\right\},\left\{\mathrm{d}_{31}, \mathrm{~d}_{32}, \mathrm{~d}_{33}\right\}\right\}, 3, " \alpha "\right]
\end{gathered}
$$

The result is

$$
\begin{gathered}
-15 d_{11}^{2} d_{13}-10 d_{11} d_{13} m_{1}^{2}-d_{13} m_{1}^{4}-8 d_{11}^{2} m_{1} m_{3}-2 d_{11} m_{1}^{3} m_{3}+4 \alpha_{1,1,1} \\
-7 d_{11} m_{1} \alpha_{2,0,1}-m_{1}^{3} \alpha_{2,0,1}+3 \alpha_{2,1,0}-2 d_{13} m_{1} \alpha_{3,0,0}
\end{gathered}
$$

Example 2.3. If we need to produce the cumulant closure of the expression $3 x_{1}^{2} x_{2}+4 x_{1} x_{2} x_{3}-x_{1}^{5} x_{3}$ to exclude moments with orders more than 3 , where $\left\{x_{1}, x_{2}, x_{3}\right\}$ is a vector with the vector $\alpha$ of mean values and the matrix $\left\{d_{i j}\right\}$ of covariances, then we may call the Cumulclosure procedure as

$$
\text { CumulClosure }\left[\left\{\mathrm{x}_{1}, \mathrm{x}_{2}, \mathrm{x}_{3}\right\}, 3 \mathrm{x}_{1}^{2} \mathrm{x}_{2}+4 \mathrm{x}_{1} \mathrm{x}_{2} \mathrm{x}_{3}-\mathrm{x}_{1}^{5} \mathrm{x}_{3}, 3, " \alpha ", " \lambda "\right]
$$


The result is

$$
\begin{gathered}
24 \alpha_{0,0,1} \alpha_{1,0,0}^{5}-30 \alpha_{1,0,0}^{4} \alpha_{1,0,1}+4 \alpha_{1,1,1} \\
-60 \alpha_{0,0,1} \alpha_{1,0,0}^{3} \alpha_{2,0,0}+60 \alpha_{1,0,0}^{2} \alpha_{1,0,1} \alpha_{2,0,0}+ \\
+30 \alpha_{0,0,1} \alpha_{1,0,0} \alpha_{2,0,0}^{2}-15 \alpha_{1,0,1} \alpha_{2,0,0}^{2}+3 \alpha_{2,1,0}-10 \alpha_{2,0,1} \alpha_{3,0,0}
\end{gathered}
$$

Example 2.4. If we need to express moments of a random vector in terms of cumulants, then we write

$$
\operatorname{Mom} \operatorname{Cum}[3,3, " \alpha ", " \lambda "]
$$

Here the first number is a dimension of this random vector and the second value is the major order of moments considered. A part of the result is the following

$$
\begin{aligned}
& \alpha_{0,0,3}=\lambda_{0,0,1}^{3}+3 \lambda_{0,0,1} \lambda_{0,0,2}+\lambda_{0,0,3} \\
& \alpha_{0,2,1}=\lambda_{0,0,1} \lambda_{0,1,0}^{2}+2 \lambda_{0,1,0} \lambda_{0,1,1}+\lambda_{0,0,1} \lambda_{0,2,0}+\lambda_{0,2,1} \\
& \alpha_{1,1,1}=\lambda_{0,0,1} \lambda_{0,1,0} \lambda_{1,0,0}+\lambda_{0,1,1} \lambda_{1,0,0}+\lambda_{0,1,0} \lambda_{1,0,1}+\lambda_{0,0,1} \lambda_{1,1,0}+\lambda_{1,1,1}
\end{aligned}
$$

Using procedure CumMom we can express cumulants of a random vector in terms of moments in the same way.

\section{Polynomial Stochastic Potentials}

Systems with polynomial coefficients of drift and diffusion arise in a lot of problems. A calculation of the steady-state PDF $p_{s}(\boldsymbol{x})$ for the system of such type can be reduced to determination of the steady-state stochastic potential $\phi(\boldsymbol{x}) \equiv-\ln p_{s}(\boldsymbol{x})$. This potential satisfies the equation

$$
\tilde{K}[\phi] \equiv \tilde{K}_{1}[\phi]+\tilde{K}_{2}[\phi]-C=0 .
$$

In a number of cases the function $\phi$ can be represented by a polynomial in the variables $x_{1}, x_{2} \ldots, x_{n}$.

Usually it is very hard to obtain an explicit form of such polynomial but some conclusions can be made.

At first, let us notice that the polynomial $\phi$ exists if

$$
\operatorname{deg}\left(\mathrm{B}_{i}\right)=\operatorname{deg}\left(a_{i}\right)=\operatorname{deg}\left(f_{i}\right), \quad \operatorname{deg}(\mathrm{C})=\operatorname{deg}\left(\sum_{i=1}^{n} \frac{\partial a_{i}}{\partial x_{i}}\right),
$$

where $\operatorname{deg}(h)=\left\{\operatorname{deg}_{1}(h), \ldots, \operatorname{deg}_{n}(h)\right\}$ and $\operatorname{deg}_{s}(h)$ denotes the degree of polynomial $h$ in $x_{s}$.

Below, on the basis of this notion we build expressions connecting the degrees of the function $\phi$ in $x_{s}$ and the coefficients of FPK Eq.(1.2). These relations allow to close a formally constructed infinite system of algebraic equations satisfied by coefficients of Maclaurin-series expansion for the potential $\phi$.

Suppose

$$
f_{i}=\sum_{\alpha=0}^{K_{i}} f_{i \alpha} x^{\alpha} \equiv \sum_{\alpha_{1}=0}^{K_{i 1}} \sum_{\alpha_{2}=0}^{K_{i 2}} \ldots \sum_{\alpha_{n}=0}^{K_{i n}} f_{i \alpha_{1} \alpha_{2} \ldots \alpha_{n}} x_{1}^{\alpha_{1}} x_{2}^{\alpha_{2}} \ldots x_{n}^{\alpha_{n}}
$$




$$
g_{i j}=\sum_{\alpha=0}^{M_{i j}} g_{i j \alpha} x^{\alpha} \equiv \sum_{\alpha_{1}=0}^{M_{i j 1}} \sum_{\alpha_{2}=0}^{M_{i j 2}} \ldots \sum_{\alpha_{n}=0}^{M_{i j n}} g_{i j \alpha_{1} \alpha_{2} \ldots \alpha_{n}} x_{1}^{\alpha_{1}} x_{2}^{\alpha_{2}} \ldots x_{n}^{\alpha_{n}}
$$

where $\alpha$ is a multi-index, $K_{i s} \geq 1, M_{i j s} \geq 0, s=1,2 \ldots, n$. To solve Eq. (3.1), let us apply the method of indeterminate coefficients and represent the function $\phi$ by the way

$$
\phi_{*}=\sum_{\alpha=0}^{N} \phi_{\alpha} x^{\alpha} \equiv \sum_{\substack{\alpha_{1}=0 \\ N_{s} \geq 2, \quad N_{\alpha_{2}=0}}}^{N_{1}} \ldots \sum_{\substack{\alpha_{n}=0 \\ s=1,2, \ldots, n}}^{N_{n}} \phi_{\alpha_{1} \alpha_{2} \ldots \alpha_{n}} x_{1}^{\alpha_{1}} x_{2}^{\alpha_{2}} \ldots x_{n}^{\alpha_{n}},
$$

Let us remark that: (i) the numbers $N_{s}$ must be an even integer; (ii) the coefficients $\phi_{\alpha}$ are equal to zero for all multi-indices $\alpha=\left\{\alpha_{1}, \alpha_{2} \ldots, \alpha_{n}\right\}$ such that $\alpha_{s} \geq N_{s}$ and $\alpha_{1}+\ldots+\alpha_{s-1}+\alpha_{s+1}+\ldots+\alpha_{n}>0(s=1,2, \ldots, n)$; (iii) $\phi_{*} \rightarrow+\infty$ as $|x| \rightarrow+\infty$.

To select correctly the degree $N$ of the polynomial $\phi_{*}$, it is necessary to establish a balance of senior degrees of polynomial $\tilde{K}\left[\phi_{*}\right]$. This polynomial is obtained after substitution of (3.3)-(3.5) in the left-hand side of Eq.(3.1). It is clear that a necessary condition of vanishing for $\tilde{K}\left[\phi_{*}\right]$ is that a coefficient at any included in $\tilde{K}\left[\phi_{*}\right]$ term of degree $N$ should be a sum of two addends at least. Thus now the main problem is to determine the degrees of polynomials $\tilde{K}_{1}\left[\phi_{*}\right]$ and $\tilde{K}_{2}\left[\phi_{*}\right]$ on the basis of $\operatorname{deg}\left(f_{i}\right), \operatorname{deg}\left(g_{i j}\right)$, and $\operatorname{deg}\left(\phi_{*}\right)$.

To solve this problem, we write the following chain of equalities

$$
\begin{gathered}
\operatorname{deg}_{s}\left(a_{i}\right)=K_{i s}, \quad \operatorname{deg}_{s}\left(b_{i j}\right)=\max _{1 \leq k \leq m}\left(M_{i k s}+M_{j k s}\right), \\
\operatorname{deg}_{s}\left(\mathrm{~A}_{i j}\right)=\operatorname{deg}_{s}\left(b_{i j}\right)=\max _{1 \leq k \leq m}\left(M_{i k s}+M_{j k s}\right), \quad \operatorname{deg}_{s}\left(\mathrm{~B}_{i}\right)=\operatorname{deg}_{s}\left(a_{i}\right)=K_{i s} .
\end{gathered}
$$

Suppose

$$
\max _{1 \leq i, j \leq n} \operatorname{deg}_{s}\left(\mathrm{~A}_{i j}\right)=2 M_{s}^{*}, \quad \max _{1 \leq i \leq n} \operatorname{deg}_{s}\left(\mathrm{~B}_{i}\right)=K_{s}^{*} .
$$

If to take into account that

$$
\begin{gathered}
\operatorname{deg}_{s}(\mathrm{C}) \leq K_{s}^{*}, \quad \operatorname{deg}_{s}\left(\frac{\partial \phi_{*}}{\partial x_{i}}\right) \leq N_{s}-1 \\
\operatorname{deg}_{s}\left(\frac{\partial^{2} \phi_{*}}{\partial x_{i} \partial x_{j}}-\frac{\partial \phi_{*}}{\partial x_{i}} \frac{\partial \phi_{*}}{\partial x_{j}}\right) \leq \operatorname{deg}_{s}\left[\frac{\partial^{2} \phi_{*}}{\partial x_{i}^{2}}-\left(\frac{\partial \phi_{*}}{\partial x_{i}}\right)^{2}\right] \leq 2 N_{s}-2,
\end{gathered}
$$

then it is possible to find

$$
\begin{gathered}
\operatorname{deg}_{s}\left(\tilde{K}_{1}\left[\phi_{*}\right]\right)=\max _{1 \leq i, j \leq n}\left[\operatorname{deg}_{s}\left(\mathrm{~A}_{i j}\right)+\right. \\
\left.+\operatorname{deg}_{s}\left(\frac{\partial^{2} \phi_{*}}{\partial x_{i} \partial x_{j}}-\frac{\partial \phi_{*}}{\partial x_{i}} \frac{\partial \phi_{*}}{\partial x_{j}}\right)\right] \leq 2 M_{s}^{*}+2 N_{s}-2,
\end{gathered}
$$




$$
\operatorname{deg}_{s}\left(\tilde{K}_{2}\left[\phi_{*}\right]\right)=\max _{1 \leq i \leq n}\left[\operatorname{deg}_{s}\left(\mathrm{~B}_{i}\right)+\operatorname{deg}_{s}\left(\frac{\partial \phi_{*}}{\partial x_{i}}\right)\right] \leq K_{s}^{*}+N_{s}-1 .
$$

Equating the right-hand sides of last inequalities, we obtain the desired result

$$
2 M_{s}^{*}+N_{s}=K_{s}^{*}+1, \quad s=1,2 \ldots, n .
$$

Example 3.1. Let the functions $f_{i}$ be linear, the coefficients $g_{i j}$ be constant $(i=1,2, \ldots, n, j=1,2, \ldots, m)$. Then $K_{s}^{*}=1, M_{s}^{*}=0$, and $N_{s}=2$. If we select proper signs of $\phi_{\alpha}$, then the result corresponds to a stochastic potential of a Gaussian type.

Example 3.2. Let us state an inverse problem, i.e., what must relations between $K_{s}^{*}$ and $M_{s}^{*}$ be for existence of a Gaussian potential? The answer following from equality $(3.6)$ is $K_{s}^{*}=2 M_{s}^{*}+1, M_{s}^{*} \geq 0$.

We have made use of this answer to construct a few stochastic systems with Gaussian potentials. The results of calculations for $\phi=\phi_{0} x^{2}$ are shown below:

$$
\begin{array}{ll}
\mathbf{M}^{*}=\mathbf{1} & f_{1}=\frac{1}{2}\left(-2 \phi_{0} g_{0}^{2}+g_{1}^{2}\right), \\
f_{0}=\frac{1}{2} g_{0} g_{1}, & f_{3}=-\phi_{0} g_{1}^{2} \\
f_{2}=-2 \phi_{0} g_{0} g_{1}, & \\
\mathbf{M}^{*}=\mathbf{2} & f_{1}=\frac{1}{2}\left(-2 \phi_{0} g_{0}^{2}+g_{1}^{2}+2 g_{0} g_{2}\right), \\
f_{0}=\frac{1}{2} g_{0} g_{1}, & f_{3}=-\phi_{0} g_{1}^{2}-2 \phi_{0} g_{0} g_{2}+g_{2}^{2}, \\
f_{2}=\frac{1}{2}\left(-4 \phi_{0} g_{0} g_{1}+3 g_{1} g_{2}\right), & f_{5}=-\phi_{0} g_{2}^{2}, \\
f_{4}=-2 \phi_{0} g_{1} g_{2}, & \\
\mathbf{M}^{*}=\mathbf{3} & f_{1}=\frac{1}{2}\left(-2 \phi_{0} g_{0}^{2}+g_{1}^{2}+2 g_{0} g_{2}\right), \\
f_{0}=\frac{1}{2} g_{0} g_{1}, & f_{3}=-\phi_{0} g_{1}^{2}-2 \phi_{0} g_{0} g_{2}+g_{2}^{2}+2 g_{1} g_{3}, \\
f_{2}=\frac{1}{2}\left(-4 \phi_{0} g_{0} g_{1}+3 g_{1} g_{2}+3 g_{0} g_{3}\right), & f_{5}=\frac{1}{2}\left(-2 \phi_{0} g_{2}^{2}-4 \phi_{0} g_{1} g_{3}+3 g_{3}^{2}\right), \\
f_{4}=\frac{1}{2}\left(-4 \phi_{0} g_{1} g_{2}-4 \phi_{0} g_{0} g_{3}+5 g_{2} g_{3}\right), & f_{7}=-\phi_{0} g_{3}^{2} . \\
f_{6}=-2 \phi_{0} g_{2} g_{3}, &
\end{array}
$$

Example 3.3. Let's construct a stochastic system with the potential $\phi=$ $\left(x_{1}^{2}+x_{2}^{2}\right)^{2}-\left(x_{1}^{2}+x_{2}^{2}\right)$ if there are only additive noises.

Here $n=2, N_{1}=N_{2}=4$. Suppose $m=2$, the matrix $G$ is diagonal, $G=\operatorname{diag}\left(g_{1}, g_{2}\right)$. Then $M_{1}^{*}=M_{2}^{*}=0$ and from equality (3.6) we obtain $K_{1}^{*}=$ $K_{2}^{*}=3$, i.e.,

$$
f_{i}=\sum_{p=0}^{3} \sum_{q=0}^{3-p} f_{i p q} x_{1}^{p} x_{2}^{q}, \quad i=1,2 .
$$

The result of calculations is as follows

$$
\begin{array}{llll}
f_{100}=0, & f_{120}=0, & f_{200}=0, & f_{220}=0, \\
f_{102}=0, & f_{130}=-2 g_{1}^{2}, & f_{202}=0, & f_{203}=-2 g_{2}^{2}, \\
f_{110}=g_{1}^{2}, & f_{101}+f_{210}=0, & f_{201}=g_{2}^{2}, & f_{103}+f_{230}=0, \\
f_{111}=0, & f_{121}+f_{230}=0, & f_{211}=0, & f_{212}-f_{230}=0, \\
f_{112}=-2 g_{1}^{2}, & & f_{221}=-2 g_{2}^{2} . &
\end{array}
$$

We see that some coefficients are indefinite. Let $C_{1}$ and $C_{2}$ be arbitrary constant values. Then the functions $f_{i}$ take the form

$$
\begin{aligned}
& f_{1}=g_{1}^{2} x_{1}-C_{1} x_{2}-2 g_{1}^{2} x_{1}\left(x_{1}^{2}+x_{2}^{2}\right)+C_{2}\left(x_{1}^{2} x_{2}+x_{2}^{3}\right), \\
& f_{2}=C_{1} x_{1}+g_{2}^{2} x_{2}-2 g_{2}^{2} x_{2}\left(x_{1}^{2}+x_{2}^{2}\right)-C_{2}\left(x_{1}^{3}+x_{1} x_{2}^{2}\right) .
\end{aligned}
$$




\section{Conclusions}

Above we have briefly described some algorithms for solution of problems, which arise during a study of effects in nonlinear dynamic systems subjected by random fluctuations, and the technique for implementation of these algorithms on the basis of the Mathematica package. The considered and some other examples such as an analysis of rotation of rigid body, an examination of stochastic systems with delay, a study of random phenomena in continuous medium, an estimation of a robot manipulator precision and so on, specify a utility and an efficiency of such implementation, especially in the frame of development of compound packages consisting of different software parts $[6$.

Acknowledgement. This research was partially supported by grants from the Russian Fund of Basic Researches ("Ural-2002" No. 02-01-96406 and No. 02-0196409).

\section{References}

1. Dimentberg, M.F.: Nonlinear Stochastic Problems of Mechanical Vibrations. Nauka, Moscow (1980)

2. Gardiner, C.W.: Handbook of Stochastic Methods for Phisics, Chemistry and the Natural Sciences. 2nd edn. Springer-Verlag, Berlin Heidelberg New York (1985)

3. Kuznetsov, P.I., Stratonovich, R.L., Tikhonov, V.I.: Quasi-moment Functions in Theory of Random Processes. DAN of USSR. 94(1954) 615-618

4. Malakhov, A.N.: Cumulant Analysis of Random Non-Gaussian Processes and Their Transforms. Sovetskoe Radio, Moscow (1978)

5. Malanin, V.V., Poloskov, I.E.: Random Processes in Nonlinear Dynamic Systems. Analytical and Numerical Methods of Analysis. Regular and Chaotic Dynamics, Ijevsk (2001)

6. Poloskov, I.E.: Compound Program Packages and a Nonlinear Random Fluctuations Analysis. In: Proc. of the 1998 Intern. Symp. on Symbolic and Algebraic Computation. ACM Press, New York (1998) 70-75

7. Pourahmadi, M.: Tailor Expansion of $\exp \left(\sum_{k=0}^{\infty} a_{k} z^{k}\right)$ and Some Applications. Amer. Math. Monthly. 91(1984) 303-307

8. Pugachev, V.S., Sinitsyn, I.N.: Stochastic Differential Systems. Nauka, Moscow (1985)

9. Wolfram, S.: The Mathematica Book. 4th edn. University Press, Cambridge (1999) 